\title{
On the root number of representations of orthogonal type
}

\author{
Erez M. Lapid \\ Dedicated to Hervé Jacquet
}

\begin{abstract}
Let $\pi$ be a generic irreducible representation of either a symplectic group or a split special even orthogonal group over a local field of characteristic zero. We prove that $\varepsilon\left(\frac{1}{2}, \pi, \psi\right)=$ $\pi(-1)$.
\end{abstract}

\section{Introduction}

Let $F$ be a local field of characteristic zero and let $G$ be either a symplectic group or a split even special orthogonal group. For any generic irreducible representation $\pi$ of $G(F)$, the $L$-factor $L(s, \pi)$ and the root number $\varepsilon(s, \pi, \psi)$ with respect to the 'standard' representation of the $L$-group of $G$ were defined by Shahidi [Sha90b]. Here $\psi$ is a fixed non-trivial character of $F$. Our main theorem is the following.

Theorem 1. For $\pi$ as above we have

$$
\varepsilon\left(\frac{1}{2}, \pi, \psi\right)=\pi(-1)
$$

This is an analogue of a result of Deligne [Del76] under the local Langlands reciprocity conjecture. Deligne proved that the triviality of the root number of a representation $\phi: W_{F} \rightarrow S O(n, \mathbb{C})$ of the Weil-Deligne group is equivalent to the possibility to lift $\phi$ to the double cover $\operatorname{Spin}(n, \mathbb{C})$ of $S O(n, \mathbb{C})$. By Langlands' conjecture, $\phi$ corresponds to a representation $\pi$ (or more precisely, an $L$-packet) of either $S p(n-1, F)$ or the split $S O(n, F)$, depending on whether $n$ is odd or even. The lifting condition on $\phi$ becomes the descent of $\pi$ to either $\operatorname{PSp}(n-1, F)$ or $\operatorname{PSO}(n, F)$ which, in turn, is equivalent to the triviality of $\pi(-1)$.

The local Langlands conjecture for $G L_{n}$ was proved not too long ago by Harris, Taylor and Henniart. It is quite deep (cf. [Car00, Rog00]), and not sufficient by itself to prove Theorem 1 (cf. [PR99]). In any case, our proof of Theorem 1 is more elementary.

Now let $k$ be a number field, $\mathbb{A}=\mathbb{A}_{k}$ its ring of adèles and $G$ a symplectic group or a split even orthogonal group over $k$. Theorem 1 immediately implies the following.

Theorem 2. Let $\Pi$ be a generic cuspidal automorphic representation of $G\left(\mathbb{A}_{F}\right)$. Then $\varepsilon\left(\frac{1}{2}, \Pi\right)=1$.

In fact, we will first prove Theorem 2 in a special case, which will imply Theorem 1 (and, hence, Theorem 2 in general). We do so by a variant of the argument of [LR03], where we use Eisenstein series on classical groups and, in particular, the inner product formula for their residues. To obtain poles we use the theta correspondence. To analyze the theta correspondence locally we use the

Received 8 April 2003, accepted in final form 7 February 2003.

2000 Mathematics Subject Classification 11F70, 11S37.

Keywords: automorphic representations, root numbers.

The author was partially supported by NSF grant DMS 0070611.

This journal is (C) Foundation Compositio Mathematica 2004. 
results of Muic and Savin [MS00]. Globally, we utilize the work of Møglin [Moe97a, Moe97b] and Ginzburg, Rallis and Soudry [GRS97].

It is an intriguing question whether it is possible to give a purely local proof of Theorem 1. The possibility of such a proof (cf. [BH99] and [LR03, Lemma 4]) is suggested by the doubling method of Piatetski-Shapiro and Rallis [PSR86]. ${ }^{1}$ A possible advantage would be to remove the condition of genericity, or even splitness from the assumptions.

The analogue of Theorem 2 for orthogonal representations of the absolute Galois group of $k$ (which follows from Deligne's result) had been proved by Frölich and Queyrut [FQ73]. There is also a result of Saito for orthogonal motives [Sai95]. On the automorphic side, several cases are discussed in [PR99].

Now let $\pi$ be a cuspidal representation of $G L_{n}(\mathbb{A})$. We say that $\pi$ is orthogonal if the symmetric square (partial) $L$-function $L^{S}\left(s, \pi, \mathrm{sym}^{2}\right)$ has a pole at $s=1$. In [GRS99], Ginzburg et al. constructed an explicit descent map from orthogonal cuspidal representations of $G L_{n}(\mathbb{A})$ with trivial central character to cuspidal generic representations of either $S p(n-1, \mathbb{A})$ or $S O(n, \mathbb{A})$, depending on whether $n$ is odd or even. At this stage, not all the expected properties of the descent map are proved (unlike the case of the descent to $S O(2 n+1)$, cf. [GRS01]). In particular, it is not clear to the author whether it is known that epsilon factors are preserved under the descent map. However, these issues are likely to be resolved in the near future. Granted the preservation of epsilon factors, we will get that $\varepsilon\left(\frac{1}{2}, \pi\right)=1$ if $\pi$ is cuspidal orthogonal with trivial central character. The central character condition would also be eliminated once the descent formalism handles the endoscopic case as well, as it does in the $S O(2 n+1)$ case. This is because root numbers of quadratic characters are known to be one. The conclusion would be a generalization (but not a new proof) of Gauss' celebrated theorem on the signs of quadratic Gauss sums. We point out that we do not expect a simple formula for the local root numbers of an orthogonal representation of $G L_{n}$ which avoids the descent.

\section{Reduction to the supercuspidal case}

Let $F$ be a local field of characteristic zero and fix a non-trivial additive character $\psi$ of $F$. For any $n$ let $S p(2 n)$ be the symplectic group with respect to

$$
\left(\begin{array}{cc}
0 & J_{n} \\
-J_{n} & 0
\end{array}\right)
$$

and let $S O(2 n)$ be the special split orthogonal group with respect to

$$
\left(\begin{array}{cc}
0 & J_{n} \\
J_{n} & 0
\end{array}\right)
$$

Here $J_{n}$ is the matrix with ones on the non-principal diagonal and zero elsewhere. We set

$$
G_{n}= \begin{cases}S p(n-1) & n \text { odd } \\ S O(n) & n \text { even }\end{cases}
$$

The $L$-group ${ }^{L} G_{n}$ of $G_{n}$ is $S O(n, \mathbb{C})$. We often denote an algebraic group and its $F$-points by the same letter. For an irreducible representation $\pi$ of $G_{n}$ we let $\omega_{\pi}$ be the scalar $\pi(-1)$. Similarly, for an irreducible representation $\pi$ of $G L_{n}$ we let $\omega_{\pi}(\cdot)$ be the central character of $\pi$. We also set $\omega_{\pi}=\omega_{\pi}(-1)$ in this case. If $\pi_{1}, \pi_{2}$ are representations of $G L_{n}, G L_{m}$, respectively, we denote by $\pi_{1} \times \pi_{2}$ the parabolically induced representation of $G L_{n+m}$ (normalized induction). Similarly, if $\tau$ is a representation of $G L_{m}$ and $\pi$ is a representation of $G_{n}$ we denote by $\tau \rtimes \pi$ the parabolically induced representation of $G_{n+2 m}$ as in [Mui01]. This is somewhat ambiguous if $n=0$, since in that

\footnotetext{
${ }^{1}$ This will appear in a forthcoming paper of the author with S. Rallis.
} 


\section{E. M. LAPID}

case there are two non-conjugate parabolic subgroups with Levi subgroup isomorphic to $G L_{m}$, but they differ by an outer involution and this ambiguity will not have any effect in what follows. We use $\times, \rtimes$ as functors, i.e. also for the induction of intertwining operators. Consider the parabolic subgroup $P=M U$ of $G_{n+2}$ whose Levi subgroup $M$ is isomorphic to $G L_{1} \times G_{n}$. The action of ${ }^{L} M$ on the Lie algebra of ${ }^{L} U$ is given by $\mathbf{1} \otimes s t d$, where $s t d$ is the 'standard' $n$-dimensional representation of ${ }^{L} G_{n}$. By Shahidi [Sha90b] we can define the local factors $L(s, \pi), \varepsilon(s, \pi, \psi), \gamma(s, \pi, \psi)$ for any irreducible generic representation $\pi$ of $G_{n}$ (with respect to some non-degenerate character of the maximal unipotent). In the case of $G L_{n}$, the same procedure applies and the local factors obtained agree with the ones defined by Godement and Jacquet in [GJ72] (cf. [Sha84]). They will also be denoted by $L(s, \pi), \varepsilon(s, \pi, \psi), \gamma(s, \pi, \psi)$. Recall that if $\pi$ is an irreducible generic representation of $G L_{n}$, then

$$
\varepsilon\left(s, \pi, \psi_{a}\right)=|a|^{n\left(s-\frac{1}{2}\right)} \omega_{\pi}(a) \varepsilon(s, \pi, \psi) .
$$

where for $a \in F^{*}$, we set $\psi_{a}(\cdot)=\psi(a \cdot)$ [Jac79]. In particular,

$$
\varepsilon\left(s, \pi, \psi^{-1}\right)=\omega_{\pi} \varepsilon(s, \pi, \psi) .
$$

Similarly, if $\pi$ is an irreducible generic representation of $G_{n}$, then

$$
\varepsilon\left(s, \pi, \psi_{a}\right)=|a|^{n\left(s-\frac{1}{2}\right)} \varepsilon(s, \pi, \psi) .
$$

This is deduced from a similar property for the gamma factor, which follows from its defining properties [Sha90b, Theorem 3.5] and the properties of the gamma factors for $G L_{1}$. In particular, the left-hand side of (1) does not depend on the choice of $\psi$. Also, since std is self-dual, we have (cf. [Sha90b, pp. 307-308])

$$
\begin{aligned}
L(s, \widetilde{\pi}) & =L(s, \pi), \\
\varepsilon(s, \widetilde{\pi}, \psi) & =\varepsilon(s, \pi, \psi) .
\end{aligned}
$$

If $\pi$ is an irreducible generic representation of either $G L_{n}$ or $G_{n}$, then

$$
\begin{gathered}
\gamma(s, \pi, \psi)=\varepsilon(s, \pi, \psi) L(1-s, \tilde{\pi}) / L(s, \pi) \\
\gamma(s, \pi, \psi) \gamma\left(1-s, \tilde{\pi}, \psi^{-1}\right)=1 \\
\varepsilon(s, \pi, \psi) \varepsilon\left(1-s, \tilde{\pi}, \psi^{-1}\right)=1
\end{gathered}
$$

[Sha90b, (3.10) and (7.4)]. If, in addition, $\pi$ is unitary, then

$$
\begin{aligned}
\overline{L(s, \pi)} & =L(\bar{s}, \tilde{\pi}) \\
\overline{\varepsilon(s, \pi, \psi)} & =\varepsilon(\bar{s}, \tilde{\pi}, \bar{\psi})
\end{aligned}
$$

[Sha90b, Proposition 7.8].

We first point out that Theorem 1 is trivial in the case $n=0,1$. For $n=2, G_{n}$ is isomorphic to a torus and if $\chi$ is a character of $G_{n}$, then

$$
\varepsilon^{S O(2)}(s, \chi, \psi)=\varepsilon^{G L_{1}}(s, \chi, \psi) \varepsilon^{G L_{1}}\left(s, \chi^{-1}, \psi\right) .
$$

Thus,

$$
\begin{aligned}
\varepsilon^{S O(2)}\left(\frac{1}{2}, \chi, \psi\right) & =\varepsilon^{G L_{1}}\left(\frac{1}{2}, \chi, \psi\right) \varepsilon^{G L_{1}}\left(\frac{1}{2}, \chi^{-1}, \psi\right) \\
& =\chi(-1) \varepsilon^{G L_{1}}\left(\frac{1}{2}, \chi, \psi\right) \varepsilon^{G L_{1}}\left(\frac{1}{2}, \chi^{-1}, \psi^{-1}\right)=\chi(-1)
\end{aligned}
$$

as required. Suppose that $\pi$ is a generic irreducible representation of $G_{n}$ with $n>2$. Then $\pi$ is a Langlands quotient of $\tau \rtimes \sigma$ where $\tau$ is a generic representation of $G L_{k}$ and $\sigma$ is a tempered generic representation of $G_{n-2 k}$. It follows from the definition of the local factors in this case [Sha90b, $\S 7$ ] that

$$
L(s, \pi)=L(s, \tau) L(s, \widetilde{\tau}) L(s, \sigma)
$$


and

$$
\varepsilon(s, \pi, \psi)=\varepsilon(s, \tau, \psi) \varepsilon(s, \widetilde{\tau}, \psi) \varepsilon(s, \sigma, \psi) .
$$

In particular, by (8) and (2) we get

$$
\begin{aligned}
\varepsilon\left(\frac{1}{2}, \pi, \psi\right) & =\varepsilon\left(\frac{1}{2}, \tau, \psi\right) \varepsilon\left(\frac{1}{2}, \widetilde{\tau}, \psi\right) \varepsilon\left(\frac{1}{2}, \sigma, \psi\right) \\
& =\varepsilon\left(\frac{1}{2}, \tau, \psi\right) \varepsilon\left(\frac{1}{2}, \widetilde{\tau}, \psi^{-1}\right) \omega_{\tau} \varepsilon\left(\frac{1}{2}, \sigma, \psi\right)=\omega_{\tau} \varepsilon\left(\frac{1}{2}, \sigma, \psi\right) .
\end{aligned}
$$

Since $\omega_{\pi}=\omega_{\tau} \omega_{\sigma}$, Theorem 1 is reduced to the tempered case.

Assume that $\pi$ is tempered and, hence, a subrepresentation of $\tau \rtimes \sigma$ where $\tau$ is tempered and $\sigma$ is square-integrable and generic. By using the multiplicativity of $L$ and $\varepsilon$-factors [Sha90a], the same argument as before reduces Theorem 1 to the square-integrable case. In particular, we are done in the case $F=\mathbb{C}$.

To reduce to the supercuspidal case we prove the following lemma.

Lemma 1. Suppose that $\pi$ is a generic square-integrable representation of $G_{n}$ which is a subrepresentation of $\pi_{1} \times \cdots \times \pi_{k} \rtimes \sigma$ where $\pi_{i}$ and $\sigma$ are supercuspidal (and generic). Then for all $i$

$$
\left.\pi_{i} \neq|\cdot|^{m-\frac{1}{2}} \quad \text { (a representation of } G L_{1}\right) \quad \text { for any } m \in \mathbb{Z} \text {. }
$$

Proof. In the case $F=\mathbb{R}$ this follows from Harish-Chandra's classification of discrete series (e.g. Proposition 1.14 of [Ada83] and, in particular, the integrality condition (1.9)). In the p-adic, we use Muic' characterization of discrete series [Mui01, Theorem 3.1]. Applying it to $\sigma$, we obtain that

$$
\gamma(s, \sigma \times \delta, \psi) \gamma\left(2 s, \delta, \wedge^{2}, \psi\right)
$$

is holomorphic with at most a simple zero at $s=0$ for any square-integrable representation $\delta$ of $G L_{r}$. Assume, on the contrary, that $m$ is the largest integer for which $\pi_{i}=|\cdot|^{ \pm\left(m-\frac{1}{2}\right)}$ for some $i$. We take $\delta$ to be the Steinberg representation of $G L_{2 m}$. Then $\gamma\left(s, \delta, \wedge^{2}, \psi\right)$ is zero at $s=0$ (e.g. [Sha92, Proposition 8.1]) and $\gamma(s, \sigma \times \delta, \psi)$ is holomorphic at $s=0$ [CS98, Theorem 4.1]. It follows that

$$
\gamma(s, \sigma \times \delta, \psi) \text { is holomorphic and non-zero at } s=0 .
$$

Similarly, applying Muic' criterion to $\pi$ (as well as [CS98, Theorem 4.1]) we obtain that

$$
\gamma(s, \pi \times \delta, \psi) \text { is holomorphic and non-zero at } s=0 .
$$

On the other hand, by the multiplicative properties of the gamma factors (cf. [MS00, Proposition 3.1]) we have

$$
\gamma(s, \pi \times \delta, \psi)=\gamma(s, \sigma \times \delta) \prod_{i} \prod_{j=-m+\frac{1}{2}}^{m-\frac{1}{2}} \gamma\left(s+j, \pi_{i}, \psi\right) \gamma\left(s+j, \tilde{\pi}_{i}, \psi\right) .
$$

For any $i, j$

$$
\gamma\left(s+j, \pi_{i}, \psi\right) \gamma\left(-s-j+1, \tilde{\pi}_{i}, \psi\right)=\omega_{\pi_{i}}
$$

by (8) and (2). From (12)-(15), it follows that $\prod_{i} \gamma\left(s, \pi_{i}, \psi\right) \gamma\left(s, \tilde{\pi}_{i}, \psi\right)$ is holomorphic and non-zero at $s=-m+\frac{1}{2}$. However, by our definition of $m, \gamma\left(s, \pi_{i}, \psi\right) \gamma\left(s, \widetilde{\pi}_{i}, \psi\right)$ is holomorphic at $s=-m+\frac{1}{2}$ for all $i$ and is zero there for at least one $i$. We obtain a contradiction.

Let $\pi, \pi_{i}, \sigma$ be as in the lemma. It follows from (11) that $\gamma\left(s, \pi_{i}, \psi\right), \gamma\left(s, \tilde{\pi}_{i}, \psi\right)$ are holomorphic at $s=\frac{1}{2}$. On the other hand, by [Sha90b, Proposition 7.2], $L(s, \pi)$ and $L(s, \sigma)$ are holomorphic for $\operatorname{Re}(s)>0$ and, in particular, at $s=\frac{1}{2}$. Thus, using (4) and (6) we have $\varepsilon\left(\frac{1}{2}, \pi, \psi\right)=\gamma\left(\frac{1}{2}, \pi, \psi\right)$ and 
similarly for $\sigma$. Again, using the multiplicativity of $\gamma$-factors we obtain

$$
\begin{aligned}
\varepsilon\left(\frac{1}{2}, \pi, \psi\right) & =\gamma\left(\frac{1}{2}, \pi, \psi\right)=\gamma\left(\frac{1}{2}, \sigma, \psi\right) \prod_{i} \gamma\left(\frac{1}{2}, \pi_{i}, \psi\right) \gamma\left(\frac{1}{2}, \tilde{\pi}_{i}, \psi\right) \\
& =\varepsilon\left(\frac{1}{2}, \sigma, \psi\right) \prod_{i} \gamma\left(\frac{1}{2}, \pi_{i}, \psi\right) \gamma\left(\frac{1}{2}, \tilde{\pi}_{i}, \psi^{-1}\right) \omega_{\pi_{i}}=\varepsilon\left(\frac{1}{2}, \sigma, \psi\right) \prod_{i} \omega_{\pi_{i}}
\end{aligned}
$$

Since $\omega_{\pi}=\omega_{\sigma} \prod_{i} \omega_{\pi_{i}}$, the reduction to the supercuspidal case follows from the subrepresentation theorems of Jacquet and Casselman. In particular, if $F$ is archimedean, Theorem 1 is proved.

Assume from now on that $F$ is $p$-adic and $\pi$ is an irreducible generic supercuspidal representation of $G_{n}, n>2$. By [Sha90b, $\S 7$ ], the local factors are unchanged if we conjugate $\pi$ by an $F$-rational element of $G_{n} / \pm 1$. Thus, we may assume that $\pi$ is $\psi$-generic, i.e. it is generic with respect to the character

$$
\chi\left(\left(x_{i, j}\right)\right)= \begin{cases}\psi\left(x_{1,2}+\cdots+x_{(n-1) / 2,(n+1) / 2}\right) & n \text { odd } \\ \psi\left(x_{1,2}+\cdots+x_{n / 2-1, n / 2}+x_{n / 2-1, n / 2+1}\right) & n \text { even. }\end{cases}
$$

In the notation of [Sha90b] this is the character $\chi_{0}$ defined by $\psi$ and the standard splitting of $G_{n}$. Note that if $n$ is even, then the property of being $\psi$-generic does not depend on the choice of $\psi$.

We now use the results of Muic and Savin [MS00] to reduce to the case where the Howe lift (i.e. local theta-lift) with respect to $\psi$ of $\pi$ to $G_{n-1}$ is zero. Indeed, suppose that the theta-lift to $G_{n-1}$ is non-zero and let $\pi^{\prime}$ be an irreducible quotient of it. Then $\pi^{\prime}$ is supercuspidal and $\psi$-generic and $\varepsilon\left(\frac{1}{2}, \pi, \psi\right)=\varepsilon\left(\frac{1}{2}, \pi^{\prime}, \psi\right)$ [MS00, Theorems 2.1 and 2.2 and Proposition 5.1]. It is also clear that $\omega_{\pi}=\omega_{\pi^{\prime}}$ because the theta-lift is defined via a homomorphism from $G_{n} \times G_{n-1}$ to a bigger symplectic group whose kernel is $Z / 2 \mathbb{Z}$ imbedded diagonally in the centers of $G_{n-1}$, $G_{n}$. Thus, for the proof of Theorem 1 we may replace $\pi$ by $\pi^{\prime}$. (Note that the theta-lift of $\pi^{\prime}$ to $G_{n-2}$ is zero by [Kud86, Theorem 2.1].)

\section{Local intertwining operators}

In this section, we make a digression and recall some facts about normalized intertwining operators. Let $F$ be a local field of characteristic zero as before and let $\pi$ be a $\psi$-generic irreducible representation of $G_{n}$. Consider the maximal parabolic subgroup $P=M U$ of $G_{n+2}$ whose Levi part is isomorphic to $G L_{1} \times G_{n}$ and the representation $I(\pi, s)=|\cdot|^{s} \rtimes \pi$ induced from $|\cdot|^{s} \otimes \pi$ (viewed as a representation of $P$ ) to $G_{n+2}$. If $n$ is even, let

$$
\epsilon_{n}=\epsilon=\left(\begin{array}{cccc}
\mathbf{1}_{n / 2-1} & & & \\
& 0 & 1 & \\
& 1 & 0 & \\
& & & \mathbf{1}_{n / 2-1}
\end{array}\right)
$$

Conjugation by $\epsilon$ defines a non-trivial outer automorphism of $G_{n}$ which preserves the standard splitting and $\chi$. For $n$ odd we set $\epsilon=1$. Let $\pi^{\epsilon}$ be the representation of $G_{n}$ on the same space as $\pi$ obtained by twisting the action by $\epsilon$. We choose a representative $w=w_{n}$ of the Weyl group of $G_{n+2}$ such that $w M w^{-1}=M$ and $w \notin M$ by the recipe given in [Sha85] with respect to the standard splitting. Specifically,

$$
w=\left(\begin{array}{lll} 
& & (-1)^{(n+1) / 2} \\
& -\mathbf{1}_{n-1} &
\end{array}\right)
$$


ON THE ROOT NUMBER OF REPRESENTATIONS OF ORTHOGONAL TYPE

if $n$ is odd and

$$
w=\left(\begin{array}{lll} 
& & (-1)^{n / 2} \\
& -\epsilon &
\end{array}\right)
$$

if $n$ is even. ${ }^{2}$ On $U$ we take the Haar measure which is self-dual with respect to $\psi$. The intertwining operator

$$
M(\pi, s): I(\pi, s) \rightarrow I\left(\pi^{\epsilon},-s\right)
$$

is defined by

$$
M(\pi, s) \varphi(g)=\int_{U} \varphi\left(w^{-1} u g\right) d u
$$

for $\operatorname{Re}(s)$ sufficiently large. It admits a meromorphic continuation in the usual sense. Shahidi defined the normalization factors

$$
m(\pi, s, \psi)=\frac{L(s, \tilde{\pi})}{\varepsilon(s, \widetilde{\pi}, \psi) L(s+1, \widetilde{\pi})} .
$$

The normalized intertwining operators $R(\pi, s, \psi)$ are given by

$$
M(\pi, s)=m(\pi, s, \psi) R(\pi, s, \psi) .
$$

If $\pi$ is unitary, then it follows from (9), (10), (4), (5) that

$$
\overline{m(\pi, s, \psi)}=m(\pi, \bar{s}, \bar{\psi})=m(\pi, \bar{s}, \psi) .
$$

Assume that $n$ is even and $\pi^{\epsilon} \simeq \pi$. Then we can define canonical local intertwining maps $\iota_{\pi}: \pi^{\epsilon} \rightarrow \pi$ by $W \mapsto W^{\epsilon}$ on the Whittaker model of $\pi$ with respect to $\psi$ where, as usual, the superscript $\epsilon$ means conjugation by $\epsilon$. By the uniqueness of the Whittaker model, $\iota_{\pi}$ does not depend on the choice of the Whittaker model. Neither does it depend on $\psi$, since upon changing $\psi$ to $\psi_{a}$, the Whittaker model is left-translated by the diagonal matrix $t=\operatorname{diag}\left(a^{n / 2-1}, \ldots, a, 1,1, a^{-1}, \ldots, a^{-n / 2+1}\right)$, and $t^{\epsilon}=t$. If $\pi$ is unramified, then $\iota_{\pi}$ fixes the unramified vector since if the conductor of $\psi$ is the ring of integers of $F$, then the unramified Whittaker function is non-zero at the identity [CS80].

For $n$ even or odd, we set

$$
\mathfrak{B}(\pi, s, \psi)=\iota_{\pi,-s} \circ R(\pi, s, \psi): I(\pi, s) \rightarrow I(\pi,-s)
$$

where $\iota_{\pi, s}=1 \rtimes \iota_{\pi}: I\left(\pi^{\epsilon}, s\right) \rightarrow I(\pi, s)$ is induced from $\iota_{\pi}$, i.e. $\iota_{\pi, s} \varphi(g)=\iota_{\pi}(\varphi(g))$. (If $n$ is odd we simply take $\iota_{\pi}=\mathbf{1}$.)

LEMma 2. Let $\pi$ be a generic irreducible representation of $G_{n}$, such that $\pi^{\epsilon} \simeq \pi$. Then we have the following.

1) $\mathfrak{B}(\pi,-s, \psi) \mathfrak{B}(\pi, s, \psi)=I$.

2) Assume that $\mathfrak{B}(\pi, s, \psi)$ (respectively $L(s, \pi)$ ) is holomorphic at $s=0$ (respectively $s=1$ ). Then $\mathfrak{B}(\pi, 0, \psi)$ has a non-trivial +1 -eigenspace (cf. $[K S 88, \S 6])$.

3) Suppose that $\pi$ is unitary. Then $\mathfrak{B}(\pi, s, \psi)^{*}=\mathfrak{B}(\pi, \bar{s}, \psi)$. Thus, $\mathfrak{B}(\pi, s, \psi)$ is unitary and, in particular, holomorphic for $\operatorname{Re}(s)=0$.

Proof. Fixing a Whittaker functional $\lambda$ on $\pi$, we define a Whittaker functional $W_{\lambda}(\pi, s)$ on $I(\pi, s)$ for $\operatorname{Re}(s) \gg 0$ by

$$
W_{\lambda}(\pi, s) \varphi=\int_{U} \lambda\left(\varphi\left(w^{-1} u\right)\right) \chi(u) d u
$$

\footnotetext{
${ }^{2}$ There is a misprint in the representatives given in [Sha02, $\left.\S 1\right]$.
} 


\section{E. M. LAPID}

It is proved in [Sha81] that $W_{\lambda}(\pi, s)$ extends to an entire function (in $s$ ) and $W_{\lambda}(\pi, s) \not \equiv 0$ for all $s$. We can view $\lambda$ as a Whittaker functional on $\pi^{\epsilon}$, since $\chi^{\epsilon}=\chi$. By definition $\lambda=\lambda \iota_{\pi}$. Thus, $W_{\lambda}\left(\pi^{\epsilon}, s\right)$ is well defined and we have

$$
W_{\lambda}\left(\pi^{\epsilon}, s\right)=W_{\lambda}(\pi, s) \iota_{\pi, s} .
$$

The local coefficients are the proportionality constants in the functional equation

$$
W_{\lambda}(\pi, s)=c(\pi, s, \psi) W_{\lambda}\left(\pi^{\epsilon},-s\right) M(\pi, s) .
$$

(They are clearly independent of $\lambda$.) By Theorem 3.5 of [Sha90b], applied to this case they are given by

$$
c(\pi, s, \psi)=\varepsilon(s, \tilde{\pi}, \bar{\psi}) L(1-s, \pi) / L(s, \tilde{\pi}) .
$$

(In fact, this relation is used to define $L, \varepsilon$.) We obtain

$$
\begin{aligned}
W_{\lambda}(\pi,-s) \mathfrak{B}(\pi, s, \psi) & =W_{\lambda}(\pi,-s) \iota_{\pi,-s} m(\pi, s, \psi)^{-1} M(\pi, s) \\
& =m(\pi, s, \psi)^{-1} W_{\lambda}\left(\pi^{\epsilon},-s\right) M(\pi, s)=m(\pi, s, \psi)^{-1} c(\pi, s, \psi)^{-1} W_{\lambda}(\pi, s) \\
& =L(1+s, \pi) / L(1-s, \pi) W_{\lambda}(\pi, s)
\end{aligned}
$$

by (4) and (3).

The first two parts of the lemma follow immediately from this relation. To prove the last part, we identify the Hermitian dual of $\pi$ with itself by choosing an invariant positive-definite inner product. We claim that $\iota_{\pi}$ is Hermitian. Indeed, since $\iota_{\pi}$ is an intertwining operator, it must preserve the inner product up to a scalar. This scalar is \pm 1 since $\iota_{\pi}$ is an involution. On the other hand, it is also positive. Hence it is one. We conclude that $\iota_{\pi, s}^{*}=\iota_{\pi^{\epsilon},-\bar{s}}$, where ${ }^{*}$ denotes the Hermitian dual. It is also easy to check the relation

$$
M\left(\pi^{\epsilon}, s\right) \iota_{\pi^{\epsilon}, s}=\iota_{\pi,-s} M(\pi, s) .
$$

Finally, $M(\pi, s)^{*}=M\left(\pi^{\epsilon}, \bar{s}\right)$. We infer that

$$
\left(\iota_{\pi,-s} M(\pi, s)\right)^{*}=M(\pi, s)^{*} \iota_{\pi,-s}^{*}=M\left(\pi^{\epsilon}, \bar{s}\right) \iota_{\pi^{\epsilon}, \bar{s}}=\iota_{\pi,-\bar{s}} M(\pi, \bar{s}) .
$$

Thus,

$$
\mathfrak{B}(\pi, s, \psi)^{*}=\mathfrak{B}(\pi, \bar{s}, \psi)
$$

by (16).

Proposition 1. Under the conditions of the previous lemma, suppose that $F$ is $p$-adic and $\pi$ is supercuspidal. Then:

1) $\mathfrak{B}(\pi, s, \psi)$ is holomorphic and not identically zero for $\operatorname{Re}(s) \geqslant 0$;

2) $\mathfrak{B}(\pi, s, \psi)$ is non-degenerate for $0 \leqslant s<1$.

If, in addition, $L(s, \pi)$ has a pole at $s=0$, then $\mathfrak{B}(\pi, 1, \psi)$ is positive semi-definite.

Proof. For $\operatorname{Re}(s)>0$, the first part follows from the corresponding statements for $M(\pi, s)$ [Sil79, Theorem 5.4.2.1] and $m(\pi, s, \psi)$ [Sha90b, Proposition 7.2, part a]. For $\operatorname{Re}(s)=0$, this follows from the previous lemma.

The second part follows from the fact that $I(\pi, s)$ is irreducible for $0<s<1$ [Sha90b, Theorem 8.1]. To prove the last part note that the condition on the $L$-function is equivalent to the irreducibility of $I(\pi, 0)$ [MS00, Lemma 6.1]. It follows from Lemma 2 that $\mathfrak{B}(\pi, 0, \psi)=\mathbf{1}$. Fix a small congruence subgroup $K$. Then on the $K$-fixed part of $I(\pi, s), \mathfrak{B}(\pi, s, \psi)$ is a continuous family of Hermitian forms for $0 \leqslant s \leqslant 1$ which is non-degenerate for $0 \leqslant s<1$ and positive-definite at $s=0$. Hence, $\mathfrak{B}(\pi, 1, \psi)$ is positive semi-definite. 


\section{The global argument}

Recall that we reduced Theorem 1 to the case where $\pi$ is a supercuspidal, irreducible generic representation of $G_{n}$ whose theta-lift to $G_{n-1}$ is zero. Assume that this is the case. Then the theta-lift $\pi^{\prime}$ of $\pi$ to $G_{n+1}$ is irreducible, generic supercuspidal and $L\left(s, \pi^{\prime}\right)$ has a pole at $s=0$ [MS00, Theorems 2.1 and 2.2 and Proposition 5.1].

Choose a totally complex number field $k$ of discriminant $D_{k}$ and a place $v_{0}$ of $k$ such that $k_{v_{0}} \simeq F$ [MS00, Lemma 5.2]. Let $\Pi=\otimes \Pi_{v}$ be a globally generic cuspidal representation of $G_{n}\left(\mathbb{A}_{k}\right)$ with respect to $\psi=\otimes \psi_{v}$ such that $\Pi_{v_{0}}=\pi$ and $\Pi_{v}$ is unramified for all finite $v$ except $v_{0}$ [Sha90b, Proposition 5.1]. We will prove Theorem 2 for this П. Since

$$
\varepsilon\left(\frac{1}{2}, \Pi\right)=\varepsilon\left(\frac{1}{2}, \pi\right) \prod_{v \mid \infty} \varepsilon\left(\frac{1}{2}, \Pi_{v}\right), \quad \omega_{\pi}=\prod_{v \mid \infty} \omega_{\Pi_{v}},
$$

and we already know Theorem 1 in the archimedean case, we will obtain Theorem 1 for $\pi$.

By our assumption on $\pi$, the theta-lift of $\Pi$ to $G_{n-1}(\mathbb{A})$ is zero (cf. [MS00]). Thus, as in [GRS97], the theta-lift of $\Pi$ to $G_{n+1}(\mathbb{A})$ is cuspidal and generic. It will soon be shown to be irreducible, but for the time being, let $\Pi^{\prime}$ be a generic irreducible constituent of it.

Let $\mathbb{E}_{\Pi}(g, \varphi, s)$ be the Eisenstein series on $G_{n+2}$ induced from $|\cdot|^{s} \otimes \Pi$. Whenever it is regular it defines an intertwining map from the induced space $I(\Pi, s)$ to the automorphic forms on $G_{n+2}(\mathbb{A})$. Let $\mathbb{E}_{\Pi^{\prime}}\left(g, \varphi^{\prime}, s\right)$ be the analogous Eisenstein series on $G_{n+3}$. Denote by $\mathfrak{M}(\Pi, s): I(\Pi, s) \rightarrow I\left(\Pi^{\epsilon_{n}},-s\right)$ the intertwining operator defined by

$$
\mathfrak{M}(\Pi, s) \varphi_{s}(g)=\int_{U(\mathbb{A})} \varphi_{s}\left(w_{n}^{-1} u g\right) d u
$$

where $w_{n}$ is as in $\S 3$. Similarly for $\mathfrak{M}\left(\Pi^{\prime}, s\right)$. By the general theory of Eisenstein series (e.g. [MW94]), it is known that $\mathbb{E}_{\Pi}(g, \varphi, s)$ and $\mathfrak{M}(\Pi, s)$ are holomorphic for $\operatorname{Re}(s)=0$ and have finitely many poles for $\operatorname{Re}(s)>0$, all of which are real and simple. They can only occur if $\Pi^{\epsilon_{n}} \simeq \Pi$. The poles of $\mathbb{E}(g, \varphi, s)$ and $\mathfrak{M}(\Pi, s)$ coincide. Similarly for $\Pi^{\prime}$.

Following Shahidi, we write the intertwining operator as

$$
\mathfrak{M}\left(\Pi^{\prime}, s\right)=m\left(\Pi^{\prime}, s\right) R\left(\Pi^{\prime}, s\right)
$$

where

$$
m\left(\Pi^{\prime}, s\right)=\frac{L\left(s, \widetilde{\Pi^{\prime}}\right)}{\varepsilon\left(s, \widetilde{\Pi^{\prime}}\right) L\left(s+1, \widetilde{\Pi^{\prime}}\right)}=\frac{L\left(s, \Pi^{\prime}\right)}{\varepsilon\left(s, \Pi^{\prime}\right) L\left(s+1, \Pi^{\prime}\right)}
$$

and $R\left(\Pi^{\prime}, s\right)=\otimes R_{v}\left(\Pi_{v}^{\prime}, s, \psi_{v}\right)$ is the 'global' normalized intertwining operator. (This is well defined since $R_{v}\left(\Pi_{v}^{\prime}, s, \psi_{v}\right)$ fixes the unramified vector for almost all $v$. As the notation suggests, $R\left(\Pi^{\prime}, s\right)$ does not depend on $\psi$.)

We claim that

and more precisely

$$
m\left(\Pi^{\prime}, s\right)=m(\Pi, s) \times \frac{\zeta_{k}(s)\left|D_{k}\right|^{s-\frac{1}{2}}}{\zeta_{k}(s+1)}
$$

$$
\begin{aligned}
L\left(s, \Pi_{v}^{\prime}\right) & =L\left(s, \Pi_{v}\right) L\left(s, \mathbf{1}_{v}\right) \\
\varepsilon\left(s, \Pi_{v}^{\prime}, \psi_{v}\right) & =\varepsilon\left(s, \Pi_{v}, \psi_{v}\right) \varepsilon\left(s, \mathbf{1}_{v}, \psi_{v}\right)
\end{aligned}
$$

for all $v$. Indeed, for $v \mid \infty$ this follows from [AB95]. For $v=v_{0}$, this follows from the main result of [MS00]. Let $v$ be a finite place different from $v_{0}$. The relation (18) follows from [Ral82]. By (3), it is enough to check (19) in the case where $\psi_{v}$ is unramified, in which case both sides are equal to one. 


\section{E. M. LAPID}

Lemma 3. The Eisenstein series $\mathbb{E}_{\Pi^{\prime}}(g, \varphi, s)$, the intertwining operator $\mathfrak{M}\left(\Pi^{\prime}, s\right)$ and the normalization factor $m\left(\Pi^{\prime}, s\right)$ have a simple pole at $s=1$. On the other hand, $\mathbb{E}_{\Pi}(g, \varphi, s), \mathfrak{M}(\Pi, s)$ and $m(\Pi, s)$ are holomorphic near $s=1$.

Proof. By [Kim02, Proposition 4.9], $R(\Pi, s)$ and $R\left(\Pi^{\prime}, s\right)$ are holomorphic and non-zero for $\operatorname{Re}(s) \geqslant 1$. Thus, by $(17)$ it is enough to check that $m(\Pi, s)$ is holomorphic and non-zero at $s=1$. If $m(\Pi, s)$ were not holomorphic at $s=1$ then $m\left(\Pi^{\prime}, s\right)$ and, hence, $\mathfrak{M}\left(\Pi^{\prime}, s\right)$ would also have at least a double pole at $s=1$, which is impossible.

By [GRS97], the partial $L$-function $L^{S}(s, \Pi)$ (with $S$ containing the archimedean places and $v_{0}$ ) is holomorphic for $\operatorname{Re}(s) \geqslant 1$ since the theta-lift of $\Pi$ to $G_{n-1}$ is zero. The same will be true for $L(s, \Pi)$ by [Kim02, Proposition 4.9].

It remains to show that $L(1, \Pi) \neq 0$. This follows from the relation (3.4) of [Sha88] and the holomorphy of $\mathbb{E}_{\Pi}(g, \varphi, s)$ at $s=0$.

Lemma 4. The Eisenstein series $\mathbb{E}_{\Pi}(g, \varphi, s)$ and intertwining operators $\mathfrak{M}(\Pi, s)$ are holomorphic for $\operatorname{Re}(s) \geqslant 0$.

Proof. Suppose that the conclusion of the lemma is false and let $s_{0}>0$ be the rightmost pole of $\mathbb{E}_{\Pi}(g, \varphi, s)$. By the main result of [Moe97a], $s_{0}$ is an integer and the theta-lift of $\Pi$ to $H$ is non-zero where $H$ is either $G_{n-2 s_{0}+1}$ or an inner form of it (if $n$ is odd). ${ }^{3}$ By Propositions 2.4 and 3.3 of [GRS97], the local Howe lift of $\Pi_{v}$ to $G_{n-2 s_{0}+1}$ is zero if $s_{0}>1$. Since $H$ splits almost everywhere, we get that $s_{0}=1$. This contradicts the previous lemma.

Since $\mathbb{E}_{\Pi^{\prime}}(g, \varphi, s)$ has a pole at $s=1$, we have $\Pi^{\prime} \simeq \Pi^{\prime \epsilon}$ where $\epsilon=\epsilon_{n+1}$. The representation $\Pi^{\prime \epsilon}$ has an automorphic realization on the space $\left\{\varphi^{\epsilon}: \varphi \in V_{\Pi^{\prime}}\right\}$ where $\varphi^{\epsilon}(g)=\varphi\left(g^{\epsilon}\right)$. We claim that the two spaces $\Pi^{\prime}$ and $\Pi^{\prime} \epsilon$ of automorphic forms on $G_{n+1} \backslash G_{n+1}(\mathbb{A})$ are 'physically' equal. Indeed, if $n$ is odd (the only non-trivial case) then the theta-lift $\Theta$ of $\Pi$ to $O(n+1, \mathbb{A})$ is cuspidal and, hence, irreducible [Moe97b]. Locally, the restriction of an irreducible representation of $O(2 r, F)$ to $S O(2 r, F)$ is either irreducible, or a sum of two inequivalent but $\epsilon$-conjugate representations. It follows that the (abstract) restriction of $\Theta$ to $S O(n+1, \mathbb{A})$ is irreducible, since it contains $\Pi^{\prime}$ and $\Pi^{\prime \epsilon} \simeq \Pi^{\prime}$. Thus, $\Theta=\Pi^{\prime}$ as spaces and, hence, $\Pi^{\prime}=\Pi^{\prime \epsilon}$ as required.

Let $\iota_{\Pi^{\prime}}: V_{\Pi^{\prime}} \rightarrow V_{\Pi^{\prime}}$ be the map $\varphi \mapsto \varphi^{\epsilon}$. Then $\iota_{\Pi^{\prime}}$ defines an intertwining map $\Pi^{\prime \epsilon} \rightarrow \Pi^{\prime}$. This is compatible with the local maps defined in $\S 3$ in the sense that $\iota_{\Pi^{\prime}}=\prod_{v} \iota_{\Pi_{v}^{\prime}}$.

We are now going to exploit the positivity of the inner product of residues of Eisenstein series as done in [LR03]. Let $E_{-1}(g, \varphi)$ (respectively $\mathfrak{M}_{-1}$ ) be the residue of $\mathbb{E}_{\Pi^{\prime}}(g, \varphi, s)$ (respectively $\left.\mathfrak{M}\left(\Pi^{\prime}, s\right)\right)$ at $s=1$. Then $E_{-1}(g, \varphi)$ is square integrable and (with an appropriate normalization of measures)

$$
\left(E_{-1}(\cdot, \varphi), E_{-1}(\cdot, \varphi)\right)=\left(\iota_{\Pi^{\prime},-1} \circ \mathfrak{M}_{-1} \varphi, \varphi\right)
$$

where on the left-hand side we take the inner product on $L^{2}\left(G_{n+3}(F) \backslash G_{n+3}(\mathbb{A})\right)$ and on the righthand side we take the pairing $I\left(\Pi^{\prime},-1\right) \times I\left(\Pi^{\prime}, 1\right) \rightarrow \mathbb{C}$. (The role of $\iota_{\Pi^{\prime}}$ is to identify $\Pi^{\prime}$ and $\Pi^{\prime \epsilon}$ through their common automorphic realization, cf. [MW94, II.1.9].) We may write $\mathfrak{M}_{-1}=$ $m_{-1} R\left(\Pi^{\prime}, 1\right)$ where $m_{-1}=\operatorname{res}_{s=1} m\left(\Pi^{\prime}, s\right)$. We conclude that

$$
\mathfrak{B}\left(\Pi^{\prime}, 1\right)=\otimes \mathfrak{B}_{v}\left(\Pi_{v}^{\prime}, 1, \psi_{v}\right)
$$

defines a semi-definite form (also denoted by $\mathfrak{B}\left(\Pi^{\prime}, 1\right)$ ) on $I\left(\Pi^{\prime}, 1\right)$, which is of the same sign as $m_{-1}$. On the other hand, by (17),

$$
m_{-1}=\frac{L(1, \Pi)}{\varepsilon(1, \Pi) L(2, \Pi)} \frac{\operatorname{res}_{s=1} \zeta_{k}(s)\left|D_{k}\right|^{\frac{1}{2}}}{\zeta_{k}(2)} .
$$

\footnotetext{
${ }^{3}$ We note the following misprint in [Moe97a, p. 203]: $\theta^{Y}(\pi \otimes \eta) \neq 0$ should be replaced by $\theta^{Y}(\pi \otimes \chi) \neq 0$.
} 
The relation (3.4) of [Sha88] together with Lemma 4 imply that $L(s, \Pi) \neq 0$ for $\operatorname{Re}(s)>1$ (cf. the proof of Proposition 4.9 in [Kim02]). We have already noted that $L(s, \Pi)$ is holomorphic for $\operatorname{Re}(s) \geqslant 1$. Since both $L(s, \pi)$ and $\varepsilon(s, \Pi)$ are real for $s \in \mathbb{R}$ and the latter is an exponential function, we conclude that the sign of $m_{-1}$ agrees with $\varepsilon\left(\frac{1}{2}, \Pi\right)$.

It remains to show that $\mathfrak{B}\left(\Pi^{\prime}, 1\right)$ is positive semi-definite. We will show that

$$
\mathfrak{B}_{v}\left(\Pi_{v}^{\prime}, 1, \psi_{v}\right) \text { is positive semi-definite }
$$

for all $v$. (We already know by Lemma 2, part 3 that $\mathfrak{B}_{v}\left(\Pi_{v}^{\prime}, 1, \psi_{v}\right)$ is Hermitian and by (20) it is semi-definite.)

For $v \neq v_{0}$ finite this is clear, since in that case $\mathfrak{B}_{v}\left(\Pi_{v}^{\prime}, s, \psi_{v}\right)$ fixes the unramified vector for all $s$. For $v=v_{0},(21)$ follows from Proposition 1 , since $\Pi_{v_{0}}^{\prime}=\pi^{\prime}$.

It remains to consider the case where $v$ is complex. Since $\Pi_{v}$ is generic, it is an irreducible principal series [Vog78]. It follows from [AB95] that $\Pi_{v}^{\prime}=\chi_{1} \times \cdots \times \chi_{[n / 2]} \rtimes \mathbf{1}$ where $\chi_{i}$ are characters of $\mathbb{C}^{*}$ and $\mathbf{1}$ denotes the trivial character of either $S O(2)$ if $n$ is odd or $S p(0)$ if $n$ is even. Since $\Pi_{v}^{\prime}$ is irreducible we may permute the $\chi_{i}$ and change any $\chi_{i}$ to $\chi_{i}^{-1}$. Since $\Pi_{v}^{\prime}$ is unitary, it is Hermitian and, hence,

$$
\left\{\chi_{1}^{ \pm 1}, \ldots, \chi_{[n / 2]}^{ \pm 1}\right\}=\left\{\bar{\chi}_{1}^{ \pm 1}, \ldots,{\overline{\chi_{[n / 2]}}}^{ \pm 1}\right\}
$$

as multi-sets. Note that if $\chi=\bar{\chi}^{-1}$ then $\chi$ is unitary, while if $\chi=\bar{\chi}$ then $\chi=|\cdot|^{\alpha}$ for some $\alpha \in \mathbb{R}$. Thus, again up to inverting some of the $\chi_{i}$, the $\chi_{i}$ consist of pairs $\kappa_{j},{\overline{\kappa_{j}}}^{-1}$ together with unitary characters and unramified characters of the form $|\cdot|^{t_{k}}$ for $t_{k} \in \mathbb{R}$. Separating the unramified $\chi_{i}$ from the ramified ones, we can write $\Pi_{v}^{\prime}$ as $\tau \rtimes \sigma$ where:

1) $\tau=\lambda_{1}|\cdot|^{\alpha_{1}} \times \lambda_{1}|\cdot|^{-\alpha_{1}} \times \cdots \times \lambda_{r}|\cdot|^{\alpha_{r}} \times \lambda_{r}|\cdot|^{-\alpha_{r}} \times \mu_{1} \times \cdots \times \mu_{s}$ where $\lambda_{i}$ and $\mu_{j}$ are unitary ramified characters of $\mathbb{C}^{*}$ and $\alpha_{i} \in \mathbb{R}_{\geqslant 0}$;

2) $\sigma=|\cdot|^{\beta_{1}} \times|\cdot|^{-\overline{\beta_{1}}} \times \cdots \times|\cdot|^{\beta_{t}} \times|\cdot|^{-\overline{\beta_{t}}} \times|\cdot|^{\gamma_{1}} \times \cdots \times|\cdot|^{\gamma_{l}} \rtimes \mathbf{1}$ where $\beta_{1}, \ldots, \beta_{t} \in \mathbb{C}$ and $\gamma_{1}, \ldots, \gamma_{l} \in$ $\mathbb{R} \cup i \mathbb{R}$.

In particular, $\tau$ and $\sigma$ are Hermitian. Since $\Pi_{v}^{\prime}$ is unitary and irreducible, both $\tau$ and $\sigma$ are unitary (cf. [Tad93, p. 20]). By the same argument, $\lambda_{i}|\cdot|^{\alpha_{i}} \times \lambda_{i}|\cdot|^{-\alpha_{i}}$ is a unitary representation for $G L_{2}(\mathbb{C})$ for any $i$. This implies that $\alpha_{i}<\frac{1}{2}$ (e.g. [Wal79]). Let $m=2 r+s$, so that $\tau$ is a representation of $G L_{m}(\mathbb{C})$.

To analyze $\mathfrak{B}_{v}\left(\Pi_{v}^{\prime}, 1, \psi_{v}\right)$, we write $w=w_{n+1}$ as $w=u_{2} w_{n+1-2 m} u_{1}$, where $w_{n+1-2 m} \in G_{n+3-2 m}$ is defined in $\S 3$ and



We view $G L_{m+1}$ and $G_{n+3-2 m}$ as subgroups of $G_{n+3}$ - the former via

$$
g \mapsto\left(\begin{array}{ccc}
g & & \\
& 1_{n+1-2 m} & \\
& & J_{m+1}{ }^{t} g^{-1} J_{m+1}
\end{array}\right)
$$

and the latter via

$$
g \mapsto\left(\begin{array}{ccc}
1_{m} & & \\
& g & \\
& & 1_{m}
\end{array}\right) .
$$

This decomposition of $w$ is reduced. There is a decomposition of $M\left(\Pi_{v}^{\prime}, s, \psi_{v}\right)$ corresponding to it 
as

$$
\left(M_{u_{2}}(-s, \tau) \rtimes \mathbf{1}\right) \circ(\mathbf{1} \rtimes M(\sigma, s)) \circ\left(M_{u_{1}}(s, \tau) \rtimes \mathbf{1}\right) .
$$

Here $M_{u_{1}}(s, \tau)$ is the intertwining operator $|\cdot|^{s} \times \tau \rightarrow \tau \times|\cdot|^{s}$ of $G L_{m+1}$ corresponding to $u_{1}$ and similarly $M_{u_{2}}(-s, \tau)$ is the intertwining operator $\tau \times|\cdot|^{-s} \rightarrow|\cdot|^{-s} \times \tau$. Note that $M_{u_{1}}(s, \tau)^{*}=$ $\omega_{\tau} M_{u_{2}}(-s, \tau)$. The factor $\omega_{\tau}$ comes from the fact that

$$
u_{2}^{-1} u_{1}=\left(\begin{array}{ll}
(-1)^{m} & \\
& -1_{m}
\end{array}\right) \text {. }
$$

Similarly, we have the factorization

$$
m\left(\Pi_{v}^{\prime}, s, \psi_{v}\right)=m\left(\tau, s, \psi_{v}\right) m\left(\widetilde{\tau}, s, \psi_{v}\right) m\left(\sigma, s, \psi_{v}\right),
$$

where

$$
m\left(\tau, s, \psi_{v}\right)=\frac{L(s, \widetilde{\tau})}{\varepsilon\left(s, \widetilde{\tau}, \psi_{v}\right) L(s+1, \widetilde{\tau})}
$$

Note that

$$
\overline{m\left(\tau, s, \psi_{v}\right)}=m\left(\widetilde{\tau}, s, \overline{\psi_{v}}\right)=\omega_{\tau} m\left(\widetilde{\tau}, s, \psi_{v}\right) .
$$

It also clear from the definition that $\iota_{\Pi_{v}^{\prime}}=\mathbf{1} \rtimes \iota_{\sigma}$. All in all,

$$
\begin{aligned}
\mathfrak{B}_{v}\left(\Pi_{v}^{\prime}, s, \psi_{v}\right)= & \left(\mathbf{1} \rtimes \iota_{\sigma,-s}\right)\left(m(\widetilde{\tau}, s, \psi)^{-1} M_{u_{2}}(-s, \tau) \rtimes \mathbf{1}\right) \\
& \times\left(\mathbf{1} \rtimes R\left(\sigma, s, \psi_{v}\right)\right)\left(m(\tau, s, \psi)^{-1} M_{u_{1}}(s, \tau) \rtimes \mathbf{1}\right) \\
= & \left(m(\widetilde{\tau}, s, \psi)^{-1} M_{u_{2}}(-s, \tau) \rtimes \mathbf{1}\right)\left(\mathbf{1} \rtimes \mathfrak{B}\left(\sigma, s, \psi_{v}\right)\right)\left(m(\tau, s, \psi)^{-1} M_{u_{1}}(s, \tau) \rtimes \mathbf{1}\right) \\
= & \left(R_{u_{1}}(s, \tau) \rtimes \mathbf{1}\right)^{*}\left(\mathbf{1} \rtimes \mathfrak{B}\left(\sigma, s, \psi_{v}\right)\right)\left(R_{u_{1}}(s, \tau) \rtimes \mathbf{1}\right)
\end{aligned}
$$

where $R_{u_{1}}(s, \tau)=m(\tau, s, \psi)^{-1} M_{u_{1}}(s, \tau)$.

We claim that $|\cdot|^{s} \times \tau$ is irreducible at $s=1$ and that $R_{u_{1}}(s, \tau)$ is holomorphic and invertible at $s=1$. The first assertion follows from the much more general Proposition I.9 of [MW89]. Here we use the fact that $\lambda_{i}, \mu_{j}$ are ramified and $\alpha_{i}<\frac{1}{2}$. (In the language of [MW89], the parameters of $\lambda_{i}$, $\mu_{j}$ are non-zero.) For the second assertion we can assume that $m=1$ by decomposing $R_{u_{1}}(s, \tau)$ into $m$ normalized intertwining operators for $G L_{2}$. The case $m=1$ follows from [MW89, Lemma I.5, part ii].

Thus, it follows from $(23)$ that $\mathfrak{B}_{v}\left(\sigma, s, \psi_{v}\right)$ is holomorphic and semi-definite at $s=1$ and its sign agrees with that of $\mathfrak{B}_{v}\left(\Pi_{v}^{\prime}, 1, \psi_{v}\right)$. This implies (21) since $\mathfrak{B}_{v}\left(\sigma, s, \psi_{v}\right)$ fixes the unramified vector.

Thus, (21) holds for all $v$ and the proof of Theorem 2 for $\Pi$ is complete.

\section{ACKNOWLEDGEMEnts}

Part of this work was done while the author was visiting Columbia University. This paper is dedicated to Hervé Jacquet for his wonderful hospitality. It is also a pleasure to thank Stephen Rallis, Freydoon Shahidi and David Soudry for useful conversations. Finally, I would to thank the referee for some helpful suggestions.

\section{REFERENCES}

AB95 J. Adams and D. Barbasch, Reductive dual pair correspondence for complex groups, J. Funct. Anal. 132 (1995), 1-42.

Ada83 J. D. Adams, Discrete spectrum of the reductive dual pair $(\mathrm{O}(p, q), \mathrm{Sp}(2 m))$, Invent. Math. 74 (1983), 449-475. 


\section{ON THE ROOT NUMBER OF REPRESENTATIONS OF ORTHOGONAL TYPE}

BH99 C. J. Bushnell and G. Henniart, Calculs de facteurs epsilon de paires pour $G L_{n}$ sur un corps local I, Bull. London Math. Soc. 31 (1999), 534-542.

Car00 H. Carayol, Preuve de la conjecture de Langlands locale pour $G L_{n}$ : travaux de Harris-Taylor et Henniart, Astérisque 266 (Exp. No. 857, 4) (2000), 191-243 (Séminaire Bourbaki, vol. 1998/99).

CS80 W. Casselman and J. Shalika. The unramified principal series of p-adic groups. II. The Whittaker function. Compositio Math. 41 (1980), 207-231.

CS98 W. Casselman and F. Shahidi, On irreducibility of standard modules for generic representations, Ann. Sci. École Norm. Sup. (4) 31 (1998), 561-589.

Del76 P. Deligne, Les constantes locales de l'équation fonctionnelle de la fonction L d'Artin d'une représentation orthogonale, Invent. Math. 35 (1976), 299-316.

FQ73 A. Fröhlich and J. Queyrut, On the functional equation of the Artin L-function for characters of real representations, Invent. Math. 20 (1973), 125-138.

GJ72 R. Godement and H. Jacquet, Zeta functions of simple algebras, Lecture Notes in Mathematics, vol. 260 (Springer, Berlin, 1972).

GRS97 D. Ginzburg, S. Rallis and D. Soudry, Periods, poles of L-functions and symplectic-orthogonal theta lifts, J. Reine Angew. Math. 487 (1987), 85-114.

GRS99 D. Ginzburg, S. Rallis and D. Soudry, On explicit lifts of cusp forms from $G L_{m}$ to classical groups, Ann. of Math. (2) 150 (1999), 807-866.

GRS01 D. Ginzburg, S. Rallis and D. Soudry, Generic automorphic forms on $\mathrm{SO}(2 n+1)$ : functorial lift to GG(2n), endoscopy, and base change, Int. Math. Res. Notices 14 (2001), 729-764.

Jac79 H. Jacquet, Principal L-functions of the linear group, in Automorphic forms, representations and L-functions, Proc. Symp. Pure Math. (Oregon State University, Corvallis, OR, 1977), Part 2 (American Mathematical Society, Providence, RI, 1979), 63-86.

Kim02 H. Kim, On local L-functions and normalized intertwining operators, Preprint (2002).

KS88 C. D. Keys and F. Shahidi, Artin L-functions and normalization of intertwining operators, Ann. Sci. École Norm. Sup. (4) 21 (1988), 67-89.

Kud86 S. S. Kudla, On the local theta-correspondence, Invent. Math. 83 (1986), 229-255.

LR03 E. Lapid and S. Rallis, On the non-negativity of $L\left(\frac{1}{2}, \pi\right)$ for $S O_{2 n+1}$, Ann. of Math. (2) 157 (2003), 891-917.

Moe97a C. Mœglin, Non nullité de certains relêvements par séries théta, J. Lie Theory 7 (1997), 201-229.

Moe97b C. Møglin, Quelques propriétés de base des séries théta, J. Lie Theory 7 (1997), 231-238.

MS00 G. Muić and G. Savin, Symplectic-orthogonal theta lifts of generic discrete series, Duke Math. J. 101 (2000), 317-333.

Mui01 G. Muić, A proof of Casselman-Shahidi's conjecture for quasi-split classical groups, Canadian Math. Bull. 44 (2001), 298-312.

MW89 C. Mœglin and J.-L. Waldspurger, Le spectre résiduel de GL(n), Ann. Sci. École Norm. Sup. (4) 22 (1989), 605-674.

MW94 C. Møglin and J.-L. Waldspurger, Décomposition spectrale et séries d'Eisenstein (Birkhäuser, Basel, 1994). Une paraphrase de l'Écriture. [A paraphrase of Scripture].

PR99 D. Prasad and D. Ramakrishnan, On the global root numbers of $G L(n) \times G L(m)$, in Automorphic forms, automorphic representations, and arithmetic (Fort Worth, TX, 1996) (American Mathematical Society, Providence, RI, 1999), 311-330.

PSR86 I. Piatetski-Shapiro and S. Rallis, $\epsilon$ factor of representations of classical groups, Proc. Natl Acad. Sci. U.S.A. 83 (1986), 4589-4593.

Ral82 S. Rallis, Langlands' functoriality and the Weil representation, Amer. J. Math. 104 (1982), 469-515.

Rog00 J. Rogawski, The nonabelian reciprocity law for local fields, Notices Amer. Math. Soc. 47 (2000), $35-41$.

Sai95 T. Saito, The sign of the functional equation of the L-function of an orthogonal motive, Invent. Math. 120 (1995), 119-142. 


\section{ON THE ROOT NUMBER OF REPRESENTATIONS OF ORTHOGONAL TYPE}

Sha81 F. Shahidi, On certain L-functions, Amer. J. Math. 103 (1981), 297-355.

Sha84 F. Shahidi, Fourier transforms of intertwining operators and Plancherel measures for GL(n), Amer. J. Math. 106 (1984), 67-111.

Sha85 F. Shahidi, Local coefficients as Artin factors for real groups, Duke Math. J. 52 (1985), 973-1007.

Sha88 F. Shahidi, On the Ramanujan conjecture and finiteness of poles for certain L-functions, Ann. Math. (2) 127 (1988), 547-584.

Sha90a F. Shahidi, On multiplicativity of local factors, in Festschrift in honor of I. I. Piatetski-Shapiro on the occasion of his sixtieth birthday, Part II (Ramat Aviv, 1989) Israel Math. Conf. Proc., vol. 3 (Weizmann, Jerusalem, 1990), 279-289.

Sha90b F. Shahidi, A proof of Langlands' conjecture on Plancherel measures; complementary series for p-adic groups, Ann. of Math. (2) 132 (1990), 273-330.

Sha92 F. Shahidi, Twisted endoscopy and reducibility of induced representations for p-adic groups, Duke Math. J. 66 (1992), 1-41.

Sha02 F. Shahidi, Local coefficients as Mellin transforms of Bessel functions: towards a general stability, Int. Math. Res. Notices 39 (2002), 2075-2119.

Sil79 A. J. Silberger, Introduction to harmonic analysis on reductive p-adic groups, in Mathematical Notes, vol. 23 (Princeton University Press, Princeton, NJ, 1979). Based on lectures by Harish-Chandra at the Institute for Advanced Study, 1971-1973.

Tad93 M. Tadić, An external approach to unitary representations, Bull. Amer. Math. Soc. (N.S.) 28 (1993), $215-252$.

Vog78 D. A. Vogan Jr., Gel'fand-Kirillov dimension for Harish-Chandra modules, Invent. Math. 48 (1978), 75-98.

Wal79 N. R. Wallach, Representations of reductive Lie groups, in Automorphic forms, representations and L-functions, Proc. Symp. Pure Math. (Oregon State University, Corvallis, OR, 1977), Part 2 (American Mathematical Society, Providence, RI, 1979), 71-86.

Erez M. Lapid erezla@math.huji.ac.il

Einstein Institute of Mathematics, The Hebrew University of Jerusalem, Jerusalem 91904, Israel 